\title{
EVALUACIÓN DE LA FRAGILIDAD BASADA EN DISTORSIÓN LATERAL PARA MUROS DE MAMPOSTERÍA CONFINADA
}

\author{
Jorge Ruiz García ${ }^{(1)}$
}

\begin{abstract}
RESUMEN
En este artículo se presentan curvas de fragilidad basadas en desplazamiento lateral desarrolladas para muros de mampostería confinada, las cuales expresan la probabilidad de que un muro de mampostería confinada alcance, o exceda, un estado de daño seleccionado dado un nivel de desplazamiento lateral. Las curvas de fragilidad fueron desarrolladas para dos estados de daño, asociados a la aparición del primer agrietamiento diagonal en la superficie de la mampostería y a la formación del agrietamiento diagonal en forma de ' $\mathrm{X}$ ', a partir de los resultados experimentales de 118 especímenes de mampostería confinada. Los especímenes fueron ensayados ante cargas del tipo sísmico, como parte de 18 programas de investigación llevados a cabo en México, Chile, Perú, Venezuela y Colombia. En particular, se incluyeron cuatro fuentes de incertidumbre asociadas a la variabilidad espécimen-a-espécimen, variabilidad de la resistencia a esfuerzo cortante de la mampostería y de la relación de aspecto de los especímenes, así como la variabilidad debido al tamaño finito de la muestra. Las curvas de fragilidad desarrolladas en este estudio son de mucha utilidad para la evaluación de la vulnerabilidad sísmica de edificaciones a base de muros de mampostería, así como para la estimación de las pérdidas económicas inducidas por eventos sísmicos.
\end{abstract}

\begin{abstract}
This paper introduces drift-based fragility curves developed for confined masonry (CM) walls, which are the main structural component for the lateral load-resisting systems employed in housing dwellings in Latin-America. The drift-based fragility curves were developed for two key damage states associated to the structural performance and reparability of CM walls from experimental results of 118 confined masonry specimens tested under lateral cyclic loading during 18 research programs carry out in Mexico, Chile, Peru, Venezuela and Colombia. In particular, four sources of uncertainty due to specimen-to-specimen, finite-sample, shear stress strength masonry and wall's aspect ratio were included. These fragility curves are very useful for assessing the seismic vulnerability of CM structures and, furthermore, for estimating the earthquake-induced economic losses employing recently proposed methodologies based on aggregating the estimated damage at the component level for a specific structure.
\end{abstract}

\section{INTRODUCCION}

Diversos eventos sísmicos históricos han evidenciado la vulnerabilidad de las edificaciones de mampostería, principalmente en aquellas ubicadas cerca de la fuente sísmica (Ruiz et al., 2003; Rodríguez, 2005; EERI, 2006). Por ejemplo, 25,353 viviendas de una muestra de 140,572 fueron

Artículo recibido el 15 de diciembre de 2007 y aprobado para su publicación el 2 de febrero de 2009. Se aceptarán comentarios y/o discusiones hasta cinco meses después de su publicación

(1) Profesor-Investigador Titular, Universidad Michoacana de San Nicolás de Hidalgo, Cd. Universitaria, Morelia 58040, e.mail: jruizgar@stanfordalumni.org 
afectadas durante el sismo de Tecomán ( $\left.\mathrm{M}_{\mathrm{w}}=7.4\right)$ de 2003 que sacudió el suroeste de la costa mexicana causando aproximadamente 123 millones de dólares en pérdidas económicas directas e indirectas (EERI, 2006). Del censo de viviendas que sufrieron daño estructural, $31.4 \%$ experimentaron daño ligero y requirieron reparaciones menores, 53.7\% exhibieron daño moderado y necesitaron reparaciones mayores, mientras que el $14.9 \%$ de las viviendas colapsaron. La ausencia de criterios ingenieriles en su construcción, dado que un alto porcentaje de las edificaciones para vivienda unifamiliar se construyen por autoconstrucción, como la ausencia de confinamiento en muros, puertas y ventanas, ha sido uno de los factores que más influyen en la vulnerabilidad sísmica de este tipo de edificaciones (Ruiz et al., 2003).

Actualmente existe un consenso entre la comunidad enfocada a la ingeniería sísmica de que el daño en elementos estructurales, y en algunos elementos no estructurales, es consecuencia de las demandas de desplazamiento lateral inducidas a la estructura cuando es expuesta a excitaciones sísmicas. En consecuencia, propuestas recientes para la evaluación de la vulnerabilidad sísmica de edificaciones existentes sugieren comparar la demanda máxima de deformación lateral a que se vería sujeta una edificación con su capacidad de deformación lateral a fin de definir su desempeño estructural. Bajo esta premisa, recientemente se han propuesto procedimientos para la evaluación de edificaciones de mampostería confinada en zonas sísmicas basados en desplazamiento lateral (Rodríguez, 2005; Terán y Zúñiga, 2007; Ruiz y Negrete, 2007).

En particular, para fines de llevar a cabo estudios de vulnerabilidad para edificaciones de mampostería confinada, así como para estimar las posibles pérdidas económicas en este tipo de edificaciones, es necesario tener una estimación del daño estructural que pudieran experimentar ante un escenario sísmico. Para tal fin, curvas de fragilidad basadas en deformación lateral para muros de mampostería confinada, las cuales expresan la probabilidad de alcanzar o exceder un estado de daño predefinido condicionado en la demanda de deformación lateral (por ejemplo, distorsión máxima de entrepiso) son una herramienta muy útil y un componente clave para estimar la vulnerabilidad sísmica de edificaciones existentes (Wen y Ellingwood, 2005). Dichas curvas de fragilidad pueden desarrollarse a partir de (Erberik y Elnashai, 2003): 1) levantamiento de daño durante inspecciones de campo después de eventos sísmicos históricos, 2) estudios analíticos de sistemas o componentes estructurales, y 3) resultados experimentales obtenidos del ensaye de especímenes sujetos a cargas del tipo sísmico.

En la actualidad, existen muy pocos estudios orientados a evaluar la fragilidad de muros de mampostería confinada. Del conocimiento del autor, el único trabajo publicado acerca de éste tópico fue desarrollado por Astroza y Schmidt (2004), los cuales desarrollaron curvas de fragilidad a partir de resultados experimentales de 52 especímenes representativos de muros de mampostería confinada ensayados en Chile, México y Venezuela. Sin embargo, debe notarse que Astroza y Schmidt (2004) solamente incluyeron en su estudio la variabilidad espécimen-a-espécimen como fuente de incertidumbre.

El objetivo del estudio que se presenta en este artículo consistió en desarrollar curvas de fragilidad basadas en deformación lateral para muros de mampostería confinada, considerando la influencia del tipo de pieza, la cuantía de refuerzo horizontal y el nivel de esfuerzo vertical de compresión actuando sobre los muros. Para ello, se recopiló una base de datos a partir de los resultados experimentales de especímenes de mampostería confinada ensayados durante diversos programas de investigación desarrollados en cinco países latinoamericanos. Asimismo, el desarrollo de las curvas de fragilidad consideró la influencia de varias fuentes de incertidumbre, como la incertidumbre inherente en las propiedades mecánicas de las piezas, representada por la resistencia a compresión diagonal de la mampostería, y en las características geométricas de los muros de mampostería, dado que los especímenes fueron ensayados con diferentes relaciones de aspecto altura-ancho. A continuación, se describe el procedimiento empleado para obtener las curvas de fragilidad basadas en deformación lateral para muros de mampostería confinada. 


\section{ESTADOS DE DAÑO SELECCIONADOS}

En principio, con base en los patrones de daño observados durante el ensaye de muros de mampostería confinada sujetos cargas de compresión diagonal o cargas cíclicas alternadas del tipo sísmico, se identificaron dos estados de daño típicos en la superficie de los muros, los cuales se pueden asociar a la aplicación de técnicas tradicionales de rehabilitación sísmica:

- Estado de daño $1\left(E D_{1}\right)$. Corresponde a la aparición de la primera grieta diagonal, normalmente menor que $0.1 \mathrm{~mm}$, en la superficie del muro. La reparación típica consiste en la inyección de mortero de cemento (Alcocer et al., 2004).

- Estado de daño $2\left(E D_{2}\right)$. Este estado de daño se caracteriza por la formación completa del agrietamiento, con anchura aproximada de $5 \mathrm{~mm}$, en forma de "X" en la superficie del muro, aplastamiento del concreto en la parte inferior de los castillos y fisuramiento horizontal distribuido en la altura de los castillos de confinamiento. Éste estado de daño coincide cuando el espécimen alcanza su capacidad máxima de carga lateral. Cuando se observa este nivel de daño, los muros de mampostería confinada requieren una combinación de técnicas de rehabilitación para restituir y mejorar sus condición original, las cuales incluyen el reemplazo de las piezas fracturadas, el rajueleo de las grietas principales y el encamisado del muro empleando malla de alambre electrosoldada recubierta con mortero de mortero de cemento (Ruiz y Alcocer, 1998; Alcocer et al., 1996; Alcocer et al., 2004).

Para fines de ilustración, en la figura 1 se muestra esquemáticamente una curva envolvente carga lateral-distorsión lateral obtenida para un muro típico de mampostería confinada y los estados de daño considerados en este estudio.

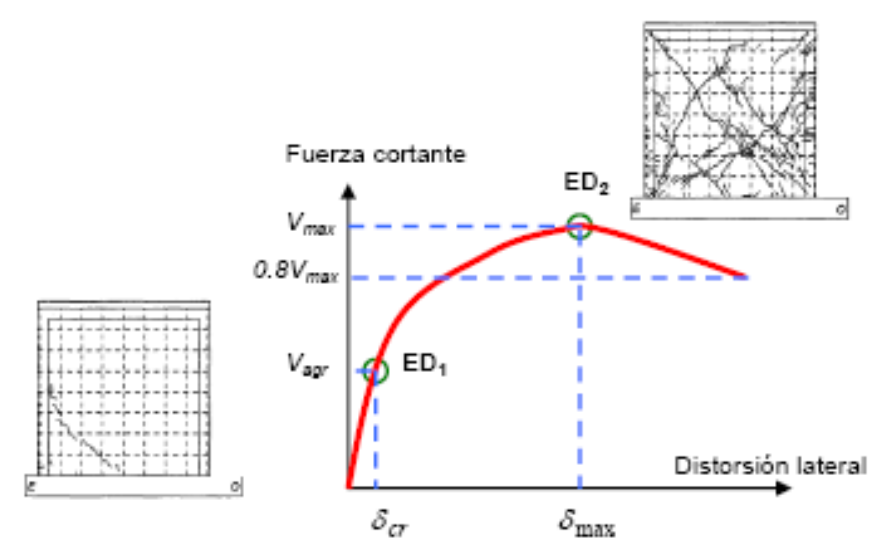

Figura 1. Estados de daño en la superficie de muros de mampostería confinada considerados en este estudio.

Debe mencionarse que la capacidad de carga lateral última de especímenes típicos de mampostería confinada (por ejemplo, correspondiente al $80 \%$ de su capacidad resistente lateral) está asociada a la progresión de la grieta diagonal hacia los castillos ocasionando plegamiento del acero longitudinal debido a esfuerzos cortantes. Sin embargo, dicho estado de daño no se consideró en esta investigación, debido a 
que frecuentemente los programas experimentales detienen los ensayos antes de que los especímenes alcancen esta condición y, en consecuencia, se dispone de un número limitado de resultados experimentales asociados a este estado de daño para llevar a cabo un estudio estadístico.

\section{BASE DE DATOS EXPERIMENTALES}

Una vez seleccionados los estados de daño de interés, se compiló una base de datos que incluye información acerca de los niveles de distorsión asociados al primer agrietamiento diagonal en la superficie del muro, $\delta_{a g r}$, así como los valores correspondientes a su capacidad lateral máxima, $\delta_{\text {max }}$, registrada durante el ensaye experimental de 118 muros de mampostería confinada sometidos ante cargas laterales cíclicas reversibles, o bien sujetos a cargas monotónicas de compresión diagonal. Los resultados experimentales fueron obtenidos de 18 programas de investigación desarrollados en México, Chile, Perú, Venezuela y Colombia. Posteriormente, los resultados experimentales fueron organizados en tres categorías de acuerdo al tipo de pieza comúnmente empleada en países latinoamericanos: a) tabique sólido de arcilla de barro rojo recocido del tipo artesanal; b) tabique sólido o hueco de arcilla del tipo industrializado; y c) bloque sólido o hueco de concreto fabricado con máquina. Asimismo, también se recopiló información adicional acerca de las propiedades mecánicas y geométricas del espécimen, como la resistencia al esfuerzo cortante de la mampostería medida en un ensaye de compresión diagonal $\left(v_{m}\right)$, la relación de aspecto del muro (es decir, altura del muro normalizada con respecto a la longitud de su base, $H / L)$, el porcentaje de acero de refuerzo horizontal $\left(\rho_{h}\right)$ y el nivel de esfuerzo vertical de compresión aplicado durante la prueba $\left(\sigma_{v}\right)$. En la tabla 1 se presenta el intervalo de valores de los parámetros mencionados anteriormente, mientras que en las tablas A1, A2 y A3 incluidas en el Apéndice A se presentan los datos experimentales correspondientes a muros de mampostería confinada construidos con cada tipo de piezas. Cabe aclarar que a pesar de un gran número de los muros de mampostería confinada fueron ensayados ante cargas laterales cíclicas, en algunas de las referencias consultadas no siempre fue posible obtener información acerca de $\delta_{\text {agr }} \mathrm{y} \delta_{\max }$ para ambos ciclos positivo y negativo y solo se consideraron los valores reportados en la referencia para conformar las bases de datos experimentales que se presentan en este estudio.

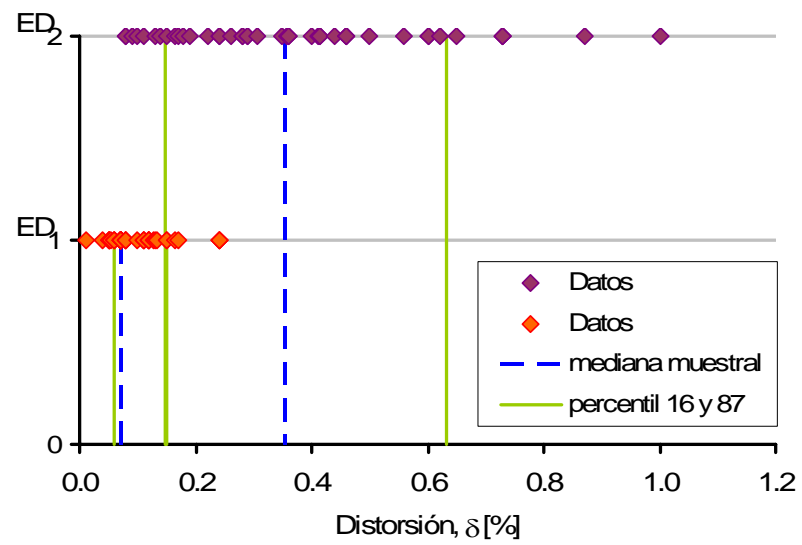

Figura 2. Distribución de distorsiones correspondientes a los estados de daño en la superficie de muros de mampostería confinada hechos con piezas artesanales de barro recocido. 
Para fines de ilustración, en la figura 2 se muestra la distribución de distorsiones correspondientes a los estados de daño seleccionados para la categoría de muros construidos con piezas de barro artesanal. Como puede observarse, existe una gran variabilidad en los valores de $\delta_{\text {agr }}$ y $\delta_{\max }$ respecto a su tendencia central (mediana muestral) para ambos estados de daño, lo cual reafirma la necesidad de emplear un enfoque probabilista para la estimación del posible estado de daño de muros de mampostería para un nivel de distorsión dado.

Tabla 1. Intervalos de valores correspondientes a los parámetros considerados en la base de datos experimentales recopilada en este estudio.

\begin{tabular}{lcccc}
\hline \multicolumn{1}{c}{ Tipo de pieza } & $\begin{array}{c}v_{m} \\
{\left[\mathrm{~kg} / \mathrm{cm}^{2}\right]}\end{array}$ & $\begin{array}{c}H / L \\
{[\mathrm{~cm} / \mathrm{cm}]}\end{array}$ & $\begin{array}{c}\rho_{h} \\
{[\%]}\end{array}$ & $\begin{array}{c}\sigma_{v} \\
{\left[\mathrm{~kg} / \mathrm{cm}^{2}\right]}\end{array}$ \\
\hline Tabique de barro recocido & $1.94-10.00$ & $0.625-1.25$ & $0-0.211$ & $0-13.92$ \\
Tabique de arcilla industrializado & $2.24-12.10$ & $0.74-1-26$ & $0-0.185$ & $0-8.60$ \\
Bloque de concreto & $2.61-7.64$ & $1.00-1-04$ & $0-0.237$ & $0-7.68$ \\
\hline
\end{tabular}

\section{CURVAS DE FRAGILIDAD BASADAS EN DISTORSION}

Para cada una de las tres categorías, de acuerdo al tipo de pieza, incluidas en la base de datos se obtuvo la distribución de probabilidad empírica acumulada de $\delta_{a g r}$ y $\delta_{\max }$ considerando los valores de distorsión de los ciclos positivos y negativos como datos independientes. Lo anterior se obtuvo al ordenar en forma ascendente los datos para cada distorsión, asignando a cada dato ordenado una probabilidad igual a $i /(n+1)$, donde $i$ es la posición del valor de distorsión y $n$ es el tamaño de la muestra (Ang y Tang, 2007). Por ejemplo, en la figura 3 se muestra la distribución empírica acumulada para ambos estados de daño correspondiente a los muros construidos con piezas de arcilla industrializada. En ambas distribuciones empíricas puede apreciarse una distribución asimétrica de los datos, $\delta$, respecto al percentil 50 (es decir, el intervalo de valores de $\delta$ es menor para probabilidades de excedencia entre $0 \%$ y $50 \%$ respecto al intervalo asociado a probabilidades de excedencia entre $50 \%$ y $100 \%$ ). Por ello, distribuciones de probabilidad asimétricas como la distribución lognormal o tipo Weibull pueden ser adecuadas para caracterizar la distribución empírica acumulada (Ang y Tang, 2007). Por ejemplo, en las figuras 3a y $3 \mathrm{~b}$ se ilustra el ajuste de ambas distribuciones paramétricas con respecto a las distribuciones empíricas para ambos estados de daño. Con el objeto de verificar si ambas distribuciones paramétricas son adecuadas para caracterizar la distribución empírica, en ésta investigación se empleó la prueba de de bondad de ajuste Kolmogorov-Smirnov (K-S) (Ang y Tang, 2007). Para fines de ilustrar gráficamente la prueba de bondad de ajuste, la distribución de probabilidad paramétrica será adecuada si todos los datos de la distribución empírica se encuentran entre las líneas de color gris incluidas en las figuras 3a y 3b. Puede verse que ambas distribuciones son adecuadas para caracterizar la probabilidad condicional de exceder un estado de daño dado un nivel de distorsión lateral. 

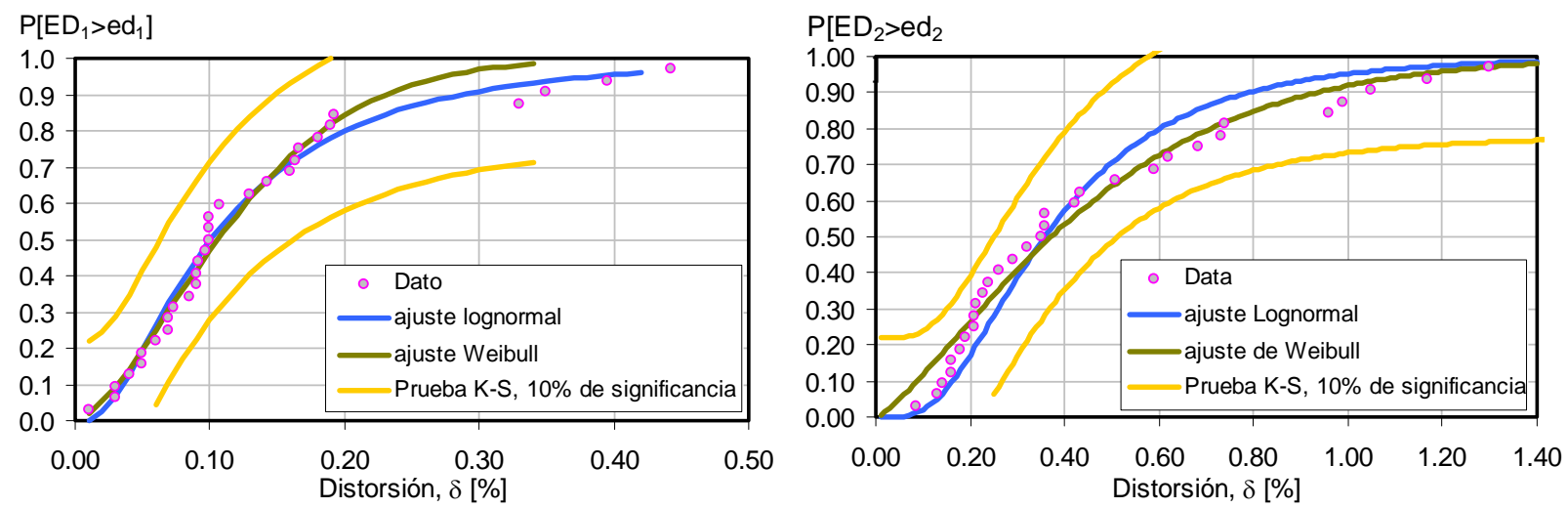

Figura 3. Comparación de curvas de fragilidad para muros de mampostería construidos con piezas de arcilla industrializada (sin refuerzo horizontal, especímenes ensayados con y sin carga vertical) obtenidas con dos funciones paramétricas (Lognormal y Weibull): a) Estado de daño 1, b) estado de daño 2.

Para fines de desarrollar una familia de curvas de fragilidad basada en distorsión, en este estudio se escogió caracterizar la fragilidad de muros de mampostería mediante una distribución paramétrica del tipo lognormal, dado que este tipo de distribución tiene la ventaja sobre otras distribuciones de incorporar explícitamente los parámetros estadísticos de tendencia central y dispersión de una muestra que solo incluye valores positivos (como son los valores de $\delta_{a g r} \mathrm{y} \delta_{\max }$ ). Este tipo de distribución paramétrica también ha sido empleada por otros investigadores durante el desarrollo de curvas de fragilidad basadas en distorsión para losas planas (Aslani y Miranda, 2005) y conexiones viga-columna (Pagni y Lowes, 2006) de concreto reforzado. Sin embargo, cabe aclarar que otro tipo de distribución paramétrica puede seleccionarse para caracterizar la fragilidad de muros de mampostería. De esta manera, la probabilidad condicional de alcanzar o exceder un estado de daño predefinido en muros de mampostería dado un nivel de distorsión lateral dado se puede expresar matemáticamente como:

$P\left(E D_{i}>e d_{i} \mid \delta\right)=1-\Phi\left(\frac{\ln (\delta)-\mu_{\ln \delta_{i}}}{\beta}\right)$

donde $P\left[E D_{i}>e d_{i} \mid \delta\right]$ es la probabilidad condicional de exceder un estado de daño $e d_{i}$ en el muro para un valor de distorsión dado $\delta$, mientras que $\mu_{\ln \delta_{i}}$ y $\beta$ son los parámetros de tendencia central y dispersión correspondientes a cada estado de daño $e d_{i}$, y $\Phi$ es la distribución acumulada normal estándar. El parámetro de dispersión $\beta$ representa la variabilidad total, la cual debería incluir tanto la incertidumbre aleatoria como epistémica (Wen et al., 2004; Dimova y Negro, 2006). La incertidumbre aleatoria se atribuye a la variabilidad inherente al fenómeno físico en estudio, mientras que la incertidumbre epistémica proviene de nuestro limitado o insuficiente conocimiento del fenómeno físico en estudio, la cual se refleja en modelos predictivos que no incluyen todas las variables inherentes a dicho fenómeno (Ang y Tang, 2007). Estudios recientes orientados al desarrollo de curvas de fragilidad basadas en evidencia experimental reconocen la existencia de diversas fuentes de incertidumbre. Por ejemplo, se considera como fuentes de incertidumbre epistémica a la cantidad limitada de datos experimentales empleados en el desarrollo de las curvas de fragilidad (Aslani y Miranda, 2005) o el uso de diversos protocolos de ensaye (Aslani y Miranda, 2005; Pagni y Lowes, 2006). Asimismo, algunas fuentes de incertidumbre aleatoria provienen de la variabilidad en las distorsiones para alcanzar un estado de daño 
dado (Wen et al., 2004; Aslani y Miranda, 2005; Pagni y Lowes, 2006), la calidad de la construcción (Dimova y Negro, 2006) y la variabilidad en las propiedades mecánicas de los especímenes (Wen et al., 2004).

En una primera etapa de esta investigación, el parámetro $\beta$ solo consideró la variabilidad en las distorsiones asociadas a cada estado de daño, la cual se conoce como variabilidad espécimen-a-espécimen $\left(\beta_{s}\right)$. En una sección por separado se describen fuentes de incertidumbre adicionales y se comenta su influencia en las curvas de fragilidad.

De esta manera, en este estudio se generó un conjunto de curvas de fragilidad basadas en distorsión para muros de mampostería confinada tomando en cuenta algunos de los parámetros que influyen en su respuesta ante cargas laterales, tales como del tipo de pieza, la cuantía de acero de refuerzo y el nivel de esfuerzo vertical de compresión . Por ejemplo, en la figura 4 se muestran las curvas de fragilidad para ambos estados de daño desarrolladas para muros de mampostería, sin acero de refuerzo horizontal, construidos con los tres tipos de piezas consideradas en este estudio. En la tabla 2 se presentan los parámetros estadísticos, para cada ensamble de datos, calculados con el método de momentos (Ang y Tang, 2007). A continuación se comenta la influencia del tipo de pieza, cuantía de acero de refuerzo y del nivel de esfuerzo vertical de compresión en las curvas de fragilidad basadas en distorsión.
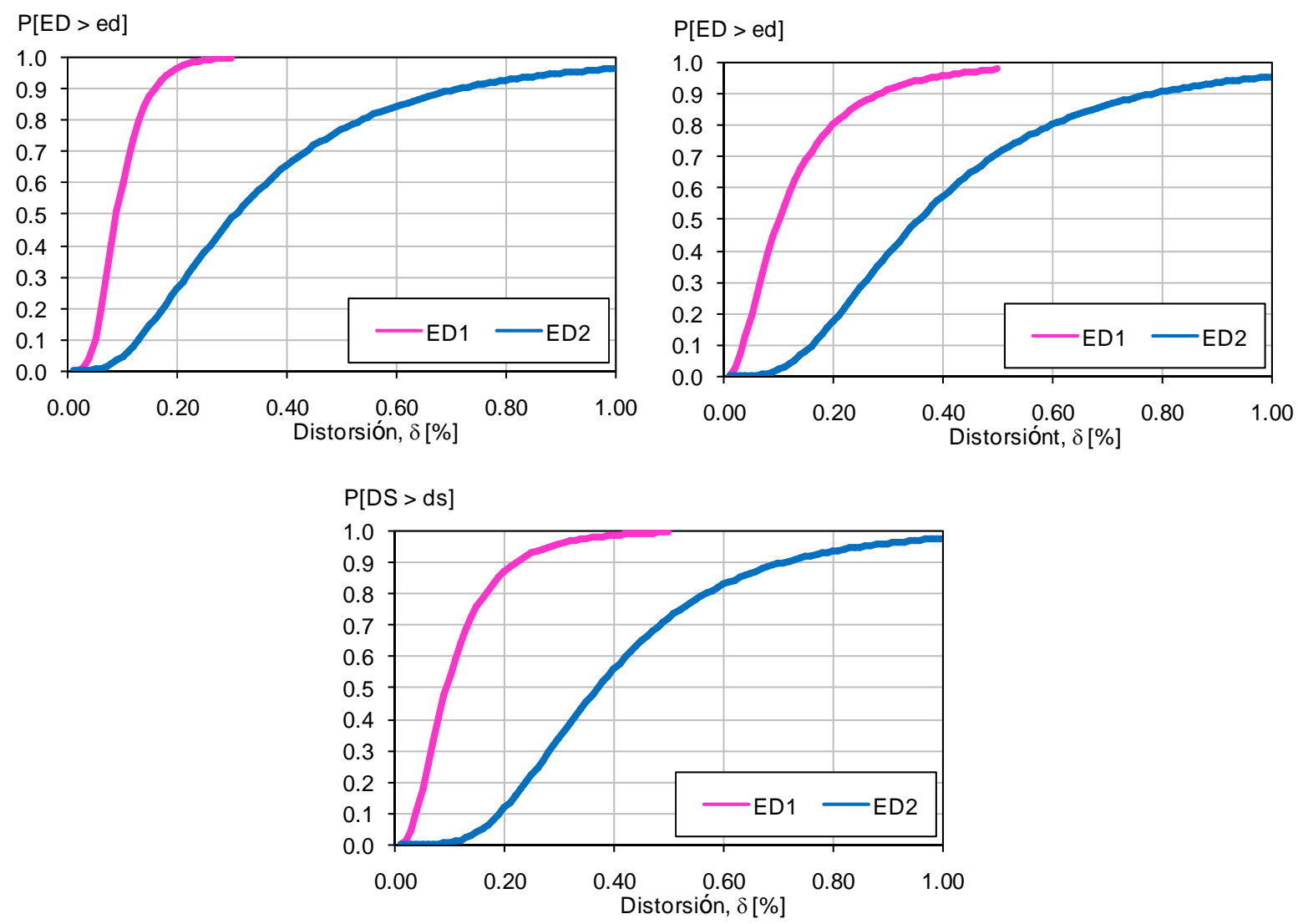

Figura 4. Curvas de fragilidad para muros de mampostería construidos con piezas artesanales (sin refuerzo horizontal, especímenes ensayados con y sin carga vertical): a) Estado de daño 1, b) estado de daño 2. 
Tabla 2. Parámetros estadísticos de tendencia central y dispersión para obtener la curva de fragilidad correspondiente a cada tipo de pieza considerada en este estudio.

\begin{tabular}{|c|c|c|c|c|c|c|}
\hline Tipo de pieza & Categoría & $\begin{array}{l}\text { Estado } \\
\text { daño }\end{array}$ & de & $\begin{array}{l}\mu_{\ln \delta} \\
(\%)\end{array}$ & $\beta_{s}$ & $\begin{array}{l}\text { Número } \\
\text { de pruebas }\end{array}$ \\
\hline \multirow{6}{*}{ Tabique de barro recocido } & \multirow[t]{2}{*}{1} & $\mathrm{ED}_{1}$ & & 0.09 & 0.46 & 44 \\
\hline & & $\mathrm{ED}_{2}$ & & 0.31 & 0.67 & 43 \\
\hline & \multirow[t]{2}{*}{2} & $\mathrm{ED}_{1}$ & & 0.17 & 0.30 & 17 \\
\hline & & $\mathrm{ED}_{2}$ & & 0.54 & 0.29 & 17 \\
\hline & \multirow[t]{2}{*}{5} & $\mathrm{ED}_{1}$ & & 0.08 & 0.38 & 14 \\
\hline & & $\mathrm{ED}_{2}$ & & 0.25 & 0.94 & 10 \\
\hline \multirow{10}{*}{ Tabique de arcilla industrializado } & \multirow[t]{2}{*}{1} & $\overline{\mathrm{ED}_{1}}$ & & 0.10 & 0.81 & 31 \\
\hline & & $\mathrm{ED}_{2}$ & & 0.36 & 0.62 & 31 \\
\hline & \multirow[t]{2}{*}{2} & $\mathrm{ED}_{1}$ & & 0.07 & 0.49 & 17 \\
\hline & & $\mathrm{ED}_{2}$ & & 0.61 & 0.62 & 17 \\
\hline & \multirow[t]{2}{*}{3} & $\mathrm{ED}_{1}$ & & 0.05 & 0.24 & 9 \\
\hline & & $\mathrm{ED}_{2}$ & & 0.61 & 0.69 & 9 \\
\hline & \multirow[t]{2}{*}{4} & $\mathrm{ED}_{1}$ & & 0.07 & 0.73 & 16 \\
\hline & & $\mathrm{ED}_{2}$ & & 0.47 & 0.81 & 16 \\
\hline & \multirow[t]{2}{*}{5} & $\mathrm{ED}_{1}$ & & 0.16 & 0.66 & 15 \\
\hline & & $\mathrm{ED}_{2}$ & & 0.27 & 0.50 & 15 \\
\hline \multirow{10}{*}{ Bloque de concreto } & \multirow[t]{2}{*}{1} & $\mathrm{ED}_{1}$ & & 0.10 & 0.68 & 64 \\
\hline & & $\mathrm{ED}_{2}$ & & 0.39 & 0.51 & 64 \\
\hline & \multirow[t]{2}{*}{2} & $\mathrm{ED}_{1}$ & & 0.11 & 0.84 & 12 \\
\hline & & $\mathrm{ED}_{2}$ & & 0.31 & 0.36 & 12 \\
\hline & \multirow[t]{2}{*}{3} & $\mathrm{ED}_{1}$ & & 0.11 & 0.87 & 9 \\
\hline & & $\mathrm{ED}_{2}$ & & 0.30 & 0.39 & 9 \\
\hline & \multirow[t]{2}{*}{4} & $\mathrm{ED}_{1}$ & & 0.09 & 0.67 & 29 \\
\hline & & $\mathrm{ED}_{2}$ & & 0.35 & 0.59 & 29 \\
\hline & \multirow[t]{2}{*}{5} & $\mathrm{ED}_{1}$ & & 0.10 & 0.68 & 35 \\
\hline & & $\mathrm{ED}_{2}$ & & 0.39 & 0.44 & 27 \\
\hline
\end{tabular}

\footnotetext{
Categoría:
}

${ }^{1}$ Especímenes con $\rho_{h}=0$ (ensayados con y sin carga vertical)

${ }^{2}$ Especímenes con acero de refuerzo horizontal (ensayados con y sin carga vertical)

${ }^{3}$ Especímenes con acero de refuerzo horizontal (ensayados sin carga vertical)

${ }^{4}$ Especímenes con $\rho_{h}=0$ y $\sigma_{v}=0$

${ }^{5}$ Especímenes con $\rho_{h}=0$ y ensayados con carga vertical.

\section{Influencia del tipo de pieza}

Para estudiar el efecto del tipo de pieza en la probabilidad de alcanzar o exceder un estado de daño y aislar la influencia del acero de refuerzo horizontal en la capacidad de deformación lateral, se desarrollaron curvas de fragilidad sin tomar en cuenta los datos muestrales de especimenes de mampostería que contenían acero de refuerzo horizontal. Sin embargo, cabe mencionar que se consideraron todos los datos provenientes de especímenes ensayados con y sin carga vertical de compresión. En las figuras 5a y 5b se muestra una comparación de las curvas de fragilidad obtenidas para cada tipo de pieza correspondientes a los estados de daño 1 y 2, respectivamente. Como puede verse en la figuras, el tipo de pieza tiene influencia en la probabilidad de alcanzar o exceder un estado de daño. Por ejemplo, existe aproximadamente una probabilidad de $50 \%$ de exceder el $E D_{2}$ a una distorsión de $0.31 \%$ 
cuando se emplea tabique artesanal, mientras que existe la misma probabilidad de exceder el $E D_{2}$ a distorsiones del orden de $0.37 \%$ cuando se emplea tabique de barro industrializado o bloque de concreto. Asimismo, a partir de las figuras, puede verse que el tipo de pieza tiene mayor influencia en las curvas de fragilidad asociadas al estado de daño 1 y 2 para probabilidades de excedencia mayores a $40 \%$ y menores a $80 \%$, respectivamente.
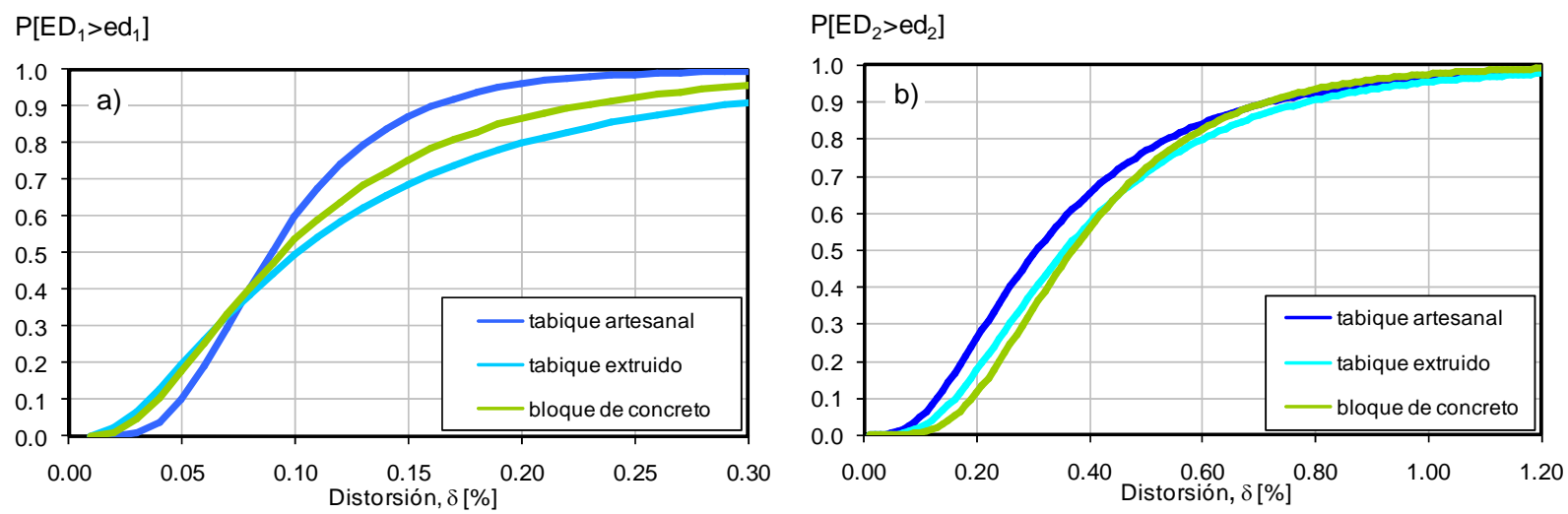

Figura 5. Influencia del tipo de pieza en las curvas de fragilidad para muros de mampostería sin refuerzo interior: a) Estado de daño 1, b) estado de daño 2.

\section{Influencia del refuerzo horizontal}

A continuación, en la figura 6 se muestran las curvas de fragilidad correspondientes a cada estado de daño obtenidas de cada ensamble de datos agrupados por el tipo de pieza considerando muros de mampostería que contienen acero de refuerzo horizontal, ensayados con y sin carga axial de compresión durante la prueba. Comparando las figuras 5 y 6 se puede apreciar la influencia del acero de refuerzo horizontal en la probabilidad de exceder los estados de daño $E D_{1}$ y $E D_{2}$. Por ejemplo, para muros de mampostería confinada construidos con tabiques artesanales, la mitad de los especímenes exceden el $E D_{1}$ a un nivel de distorsión de $0.17 \%$ cuando se incluye acero de refuerzo horizontal mientras que la mitad de los especímenes exceden el mismo estado de daño a una distorsión de $0.09 \%$ cuando no se incluye el refuerzo horizontal. Asimismo, la mitad de los especímenes excederán el $E D_{2}$ a un nivel de distorsión del orden de $0.31 \%$ cuando no incluyen acero de refuerzo horizontal, mientras que excederán el mismo estado de daño a una distorsión de $0.56 \%$ cuando se incluye acero de refuerzo horizontal.

\section{Influencia del esfuerzo vertical de compresión}

Con el objeto de investigar si el esfuerzo vertical de compresión actuante durante la prueba tiene alguna influencia en la probabilidad de excedencia de los estados $E D_{1}$ y $E D_{2}$ se desarrollaron curvas de fragilidad considerando especímenes ensayados sin y con carga axial durante la prueba. Dicha influencia se estudió para especímenes sin y con acero de refuerzo horizontal. De esta manera, en la figuras 7a y 7b se muestra una comparación entre las curvas de fragilidad correspondientes los estados de daño 1 y 2 , respectivamente, obtenidas de muros de muros de mampostería confinada, sin refuerzo horizontal entre las hiladas, construidos con tabique de arcilla industrializado, con y sin carga vertical aplicada durante la prueba. Para este tipo de muros, el esfuerzo vertical de compresión aplicado a los especímenes se encuentra en un intervalo de 1.76 a $8.6 \mathrm{~kg} / \mathrm{cm}^{2}$, el cuál es representativo del nivel de carga axial en muros de la planta baja de edificaciones de 3 a 5 niveles construidos en México. A partir de la figuras, se puede observar que la presencia del esfuerzo vertical durante el ensaye tiene influencia importante en las curvas 
de fragilidad. Por ejemplo, se puede apreciar que la mitad de los especímenes excederán el $E D_{2}$ a una distorsión del orden de $0.48 \%$ en ausencia del esfuerzo vertical de compresión mientras que la misma cantidad de especímenes excederán el mismo estado de daño a una distorsión aproximada de $0.27 \%$ en presencia de esfuerzo vertical. Por otro lado, cabe notar que la probabilidad de excedencia del mismo estado de daño 2 se incrementa cuando actúa carga vertical en los muros. Por ejemplo, si un muro de mampostería se sujeta a una distorsión lateral de $0.40 \%$ existe un $42 \%$ y $80 \%$ de probabilidad de que alcance este estado de daño en ausencia y presencia de carga vertical. Cabe notar que la tendencia mostrada en las curvas de fragilidad refleja la disminución de la capacidad de desplazamiento lateral desde la aparición del primer agrietamiento hasta la formación de agrietamiento asociado a la resistencia lateral en presencia de la carga vertical de compresión para muros construidos con tabique industrializado que ha sido notada por otros autores (Zepeda et al., 2000). No obstante las observaciones anteriores, cuando los muros de mampostería cuentan con refuerzo horizontal no se aprecia una influencia importante de la carga axial, como puede apreciarse en la figura 8.
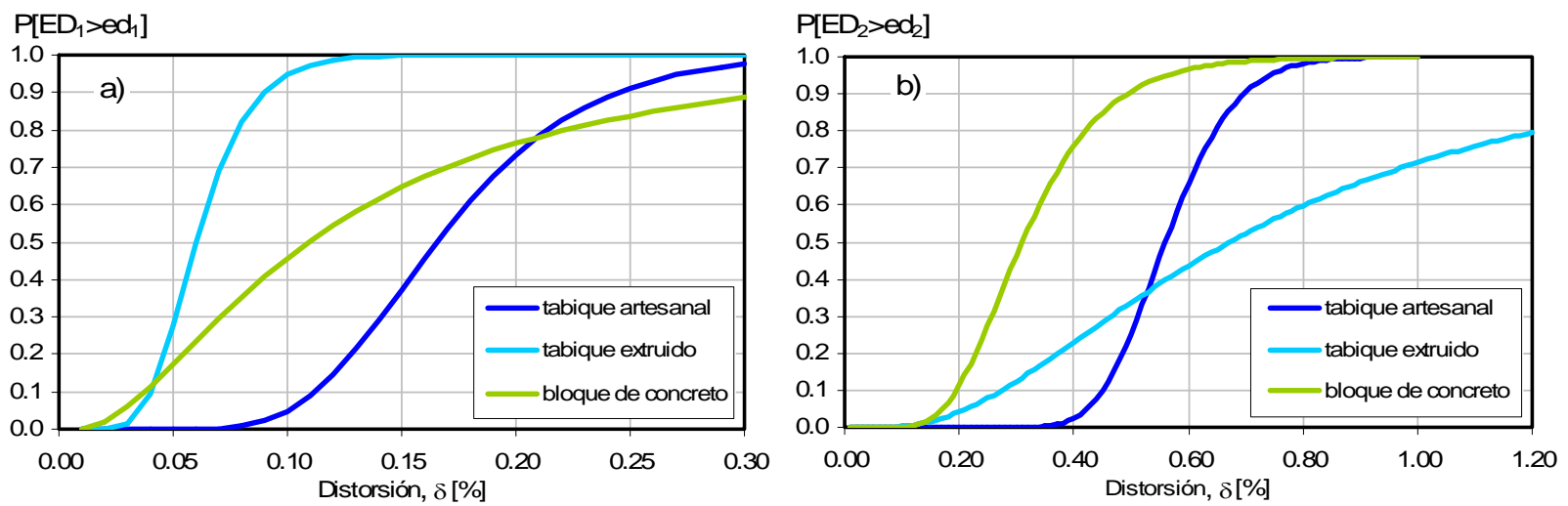

Figura 6. Influencia del tipo de pieza en las curvas de fragilidad para muros de mampostería con refuerzo horizontal interior: a) Estado de daño 1, b) estado de daño 2.
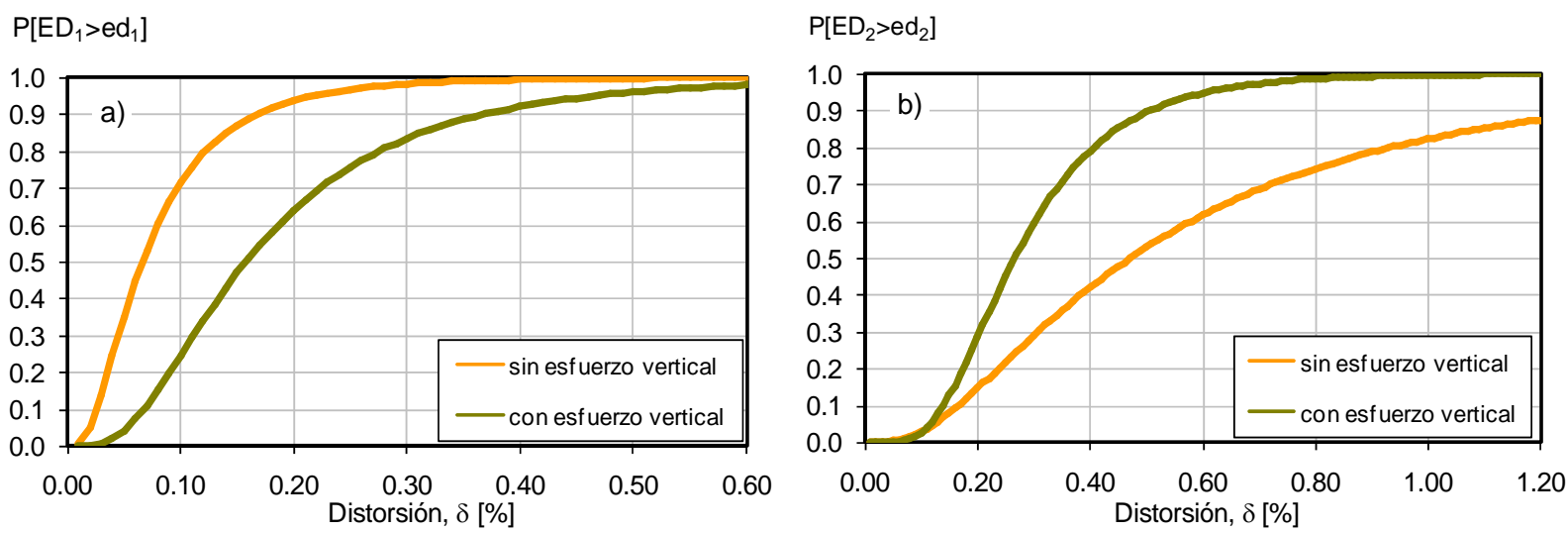

Figura 7. Influencia del nivel de carga axial en las curvas de fragilidad para muros de mampostería construidos con tabique de arcilla industrializado (sin incluir acero de refuerzo horizontal):

a) Estado de daño 1, b) estado de daño 2. 
Asimismo, la influencia de la carga axial en las curvas de fragilidad parece ser menos significativa en muros construidos con bloque de concreto, sin y con refuerzo horizontal, respecto a los muros construidos con tabique de arcilla industrializado. Por ejemplo, en la figuras 9a y 9b se muestra una comparación entre las curvas de fragilidad obtenidas para muros sin acero de refuerzo horizontal, con y sin esfuerzo vertical, para ambos estados de daño. Desafortunadamente, la influencia de la carga vertical en muros construidos con tabique de barro recocido no pudo evaluarse debido a la falta de resultados experimentales para desarrollar las curvas de fragilidad.
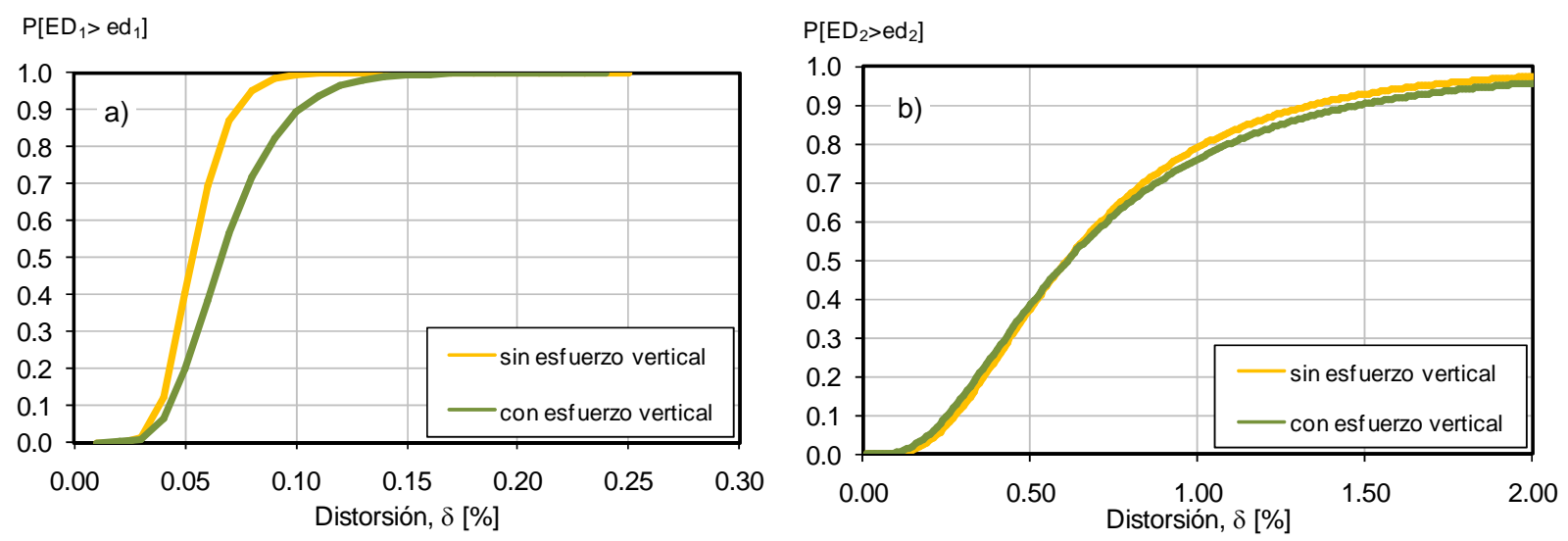

Figura 8. Influencia del nivel de carga axial en las curvas de fragilidad para muros de mampostería construidos con tabique de arcilla industrializado con acero de refuerzo horizontal:

a) Estado de daño 1, b) estado de daño 2.
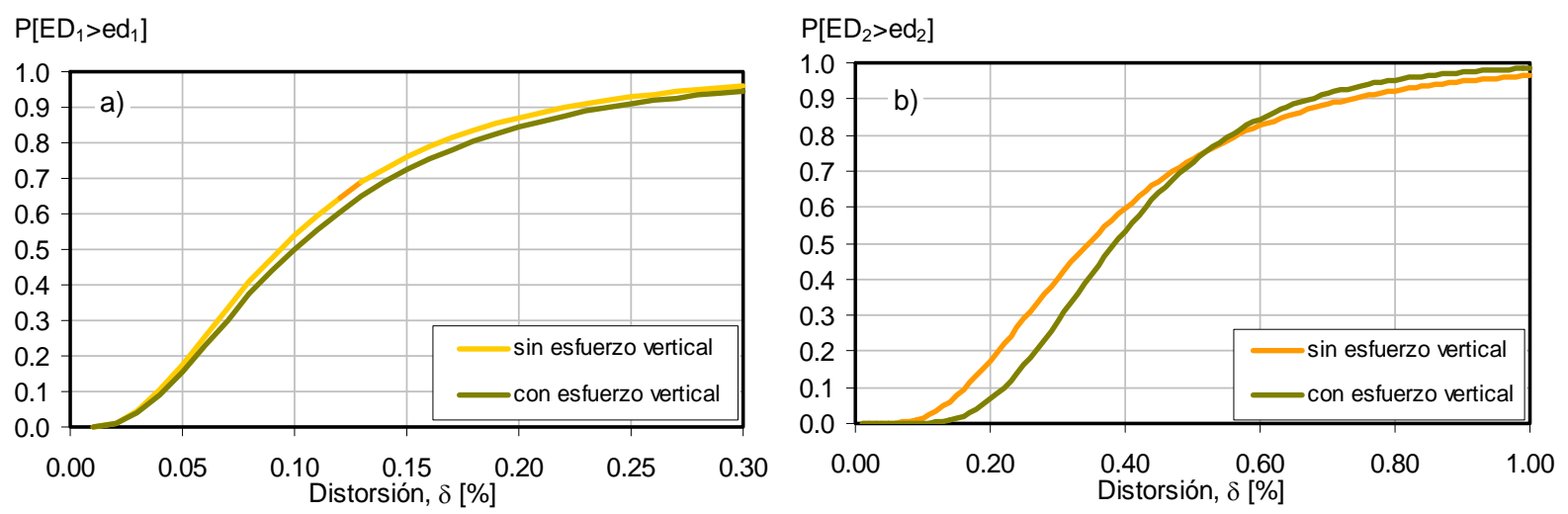

Figura 9. Influencia del nivel de carga axial en las curvas de fragilidad para muros de mampostería construidos con bloque de concreto: a) Estado de daño 1, b) estado de daño 2.

\section{Influencia de fuentes de incertidumbre adicionales en las curvas de fragilidad}

Como se comentó anteriormente, en el desarrollo de las curvas de fragilidad se debería considerar la variabilidad total, la cual incluye la incertidumbre aleatoria, $\beta_{a}$, y epistémica, $\beta_{e}$. De acuerdo a Ang y Tang (2007), la influencia de la incertidumbre aleatoria debería considerarse en la estimación de la probabilidad condicional de alcanzar un estado de daño dada en la ec. (1), mientras que en la incertidumbre epistémica debería expresarse a través de la variabilidad de la probabilidad estimada con la 
ec. (1). Este criterio fue adoptado por Aslani y Miranda (2005) para desarrollar curvas de fragilidad basada en distorsión para losas planas de concreto reforzado. Sin embargo, algunos investigadores sugieren considerar explícitamente la variabilidad total en la ec. (1), al combinar ambas fuentes de incertidumbre como $\beta=\sqrt{\beta_{a}{ }^{2}+\beta_{e}{ }^{2}}$, suponiendo que $\beta_{a}$ y $\beta_{e}$ no están correlacionadas (Wen et al., 2004; Dimova y Negro, 2006).

Para fines de esta investigación, se adoptó el criterio de Ang y Tang (2007) y se consideraron las siguientes fuentes de incertidumbre adicionales a la variabilidad espécimen-a-espécimen: a) incertidumbre aleatoria debida a diferencias en la geometría de los especímenes de mampostería confinada, $\beta_{h}$, caracterizadas por la relación de aspecto de los muros, b) incertidumbre aleatoria debida a la variabilidad en las propiedades mecánicas de los especímenes, representada por la resistencia a esfuerzo cortante de la mampostería, $\beta_{v}$, así como c) incertidumbre epistémica, debido al número limitado de resultados experimentales empleados en el desarrollo de las curvas de fragilidad. Cabe notar que todas las fuentes de incertidumbre fueron calculadas a partir de la desviación estándar del logaritmo de los datos. En la tabla 3 se presentan los valores calculados de las fuentes de incertidumbre $\beta_{s}, \beta_{h}$ y $\beta_{v}$ para cada categoría en la base de datos.

Tabla 3. Fuentes adicionales de incertidumbre, debido a la resistencia al esfuerzo cortante y a la relación de aspecto, incluidas en este estudio (calculada como la desviación estándar logarítmica).

\begin{tabular}{lcc}
\hline \multicolumn{1}{c}{ Tipo de pieza } & $\begin{array}{c}\text { Resistencia al } \\
\text { esfuerzo cortante }\left(v_{m}\right),\end{array}$ & $\begin{array}{c}\text { Relación de } \\
\text { aspecto (H/L), }\end{array}$ \\
& $\beta_{v}$ & $\beta_{h}$ \\
\hline Tabique de barro recocido & 0.479 & 0.224 \\
Tabique de arcilla industrializado & 0.428 & 0.026 \\
Bloque de concreto & 0.255 & 0.110 \\
\hline
\end{tabular}

De esta manera, suponiendo que las fuentes de incertidumbre $\beta_{s}, \beta_{h} \mathrm{y} \beta_{v}$ no están estadísticamente correlacionadas, el parámetro $\beta$, que incorpora la incertidumbre aleatoria, se puede obtener de la siguiente manera:

$\beta=\sqrt{\beta_{s}^{2}+\beta_{h}{ }^{2}+\beta_{v}^{2}}$

La incertidumbre epistémica debido a número limitado de datos se consideró mediante intervalos de confianza para $\beta$, como lo sugieren (Ang y Tang, 2007; Aslani y Miranda, 2005):

$\left[\frac{(n-1) \cdot \beta^{2}}{\chi^{2} \alpha / 2, n-1}\right]^{\frac{1}{2}}$ y $\left[\frac{(n-1) \cdot \beta^{2}}{\chi^{2}{ }_{1-\alpha / 2, n-1}}\right]^{\frac{1}{2}}$

donde $\chi_{\alpha / 2, n-1}^{2}$ es la inversa de la distribución $\chi^{2}$ con $n-1$ grados de libertad para un nivel de significancia $\alpha$, mientras que $\chi^{2}{ }_{1-\alpha / 2, n-1}$ es la inversa de la distribución $\chi^{2}$ teniendo $n-1$ grados de libertad con un nivel de significancia $1-\alpha$. Finalmente, se tomaron en cuenta todas las fuentes de 
incertidumbre identificadas en este estudio en los parámetros de tendencia central de las distorsiones asociadas a cada estado de daño por medio de los intervalos de confianza (e.g. con un nivel de significancia del 5\%) como lo recomiendan Ang y Tang (2007):

$\mu_{\ln } \delta_{i} \cdot \exp \left[ \pm 1.96 \frac{\beta}{\sqrt{n}}\right]$

donde $n$ es el tamaño de la muestra y $\beta$ se calcula a partir de la ec. (2).

Cabe notar que existen otras fuentes de incertidumbre que deberían tomarse en consideración, tales como la incertidumbre en la calidad de la construcción, dado que en la práctica las edificaciones a base de muros de mampostería confinada se construyen sin supervisión profesional (Dimova y Negro, 2006). Sin embargo, por el momento, no existe información experimental disponible para incluir dicha fuente de incertidumbre.

A fin de ilustrar la influencia de las fuentes adicionales de incertidumbre, en la figura 10 se presentan las curvas de fragilidad para ambos estados de daño obtenidas para muros de mampostería construidos con tabique artesanal, sin incluir acero de refuerzo horizontal, considerando solo la variabilidad espécimen-a-espécimen y las fuentes adicionales de incertidumbre (obtenidas para un 5\% de significancia). Puede observarse que es importante incluir las fuentes adicionales de incertidumbre dado que la probabilidad de excedencia de los estados de daño puede cambiar significativamente. Por ejemplo, en la figura $11 \mathrm{~b}$ se puede observar que existe un $50 \%$ de probabilidad de que aparezcan grietas diagonales en forma de " $X$ " en la superficie del muro (estado de daño 2) para un nivel de distorsión entre $0.30 \%$ y $0.42 \%$. Asimismo, debe notarse que la probabilidad de excedencia de un estado de daño cambia al incluirse las fuentes adicionales de incertidumbre. Por ejemplo, la probabilidad de exceder el $E D_{2}$ varía de $48 \%$ a $65 \%$ cuando los muros de mampostería son sujetos a un nivel de distorsión del orden de $0.40 \%$, mientras que incluyendo solamente la variabilidad espécimen-a-espécimen se observa que existe únicamente una probabilidad de 57\% de excedencia.
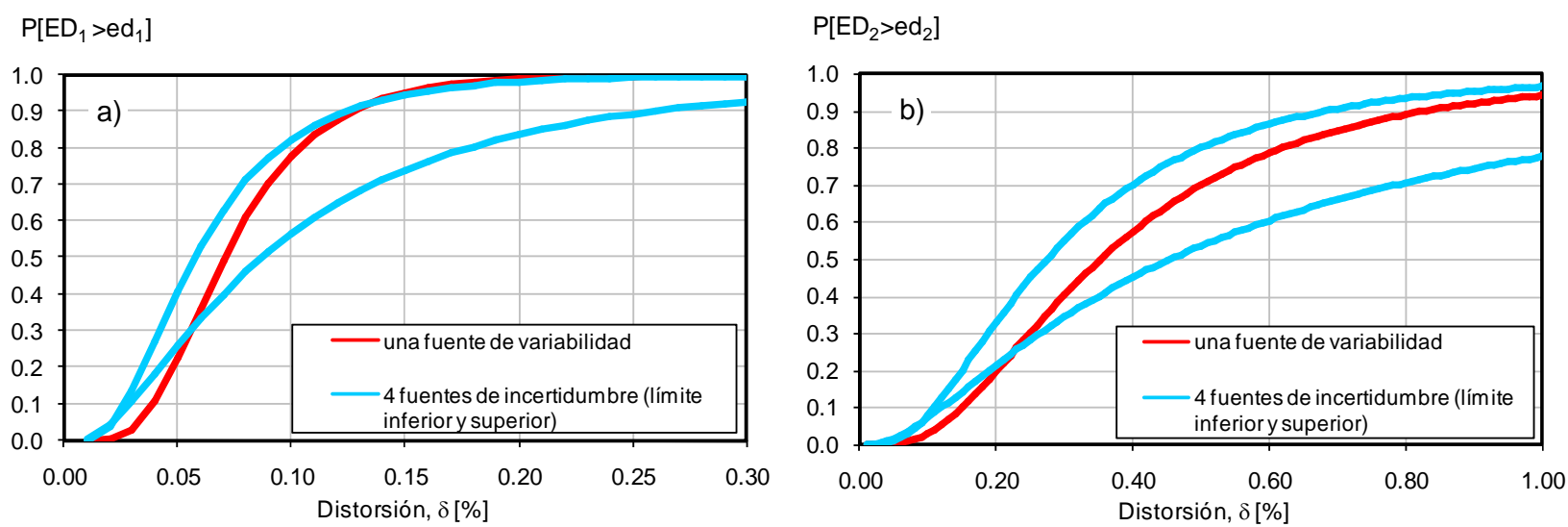

Figura 10. Influencia de fuentes de incertidumbre en las curvas de fragilidad para muros de mampostería construidos con tabique artesanal (sin refuerzo horizontal interior): a) Estado de daño 1, b) estado de daño 


\section{COMENTARIOS SOBRE LOS LÍMITES PERMISIBLES DE DISTORSIÓN DE ENTREPISO PARA MUROS DE MAMPOSTERÍA}

Como se ha mencionado anteriormente, a fin de establecer metodologías de diseño y evaluación sísmica basadas en conceptos de desempeño, es necesario establecer límites de desplazamiento lateral (o distorsión lateral) asociados a varios estados de daño. Para edificaciones a base de muros de mampostería, dichos límites de distorsión deberían considerar las variables que influyen en la capacidad de deformación. Por ejemplo, en el Apéndice A de la Normas Técnicas Complementarias para el Diseño por Sismo Edición 2004 (NTS, 2004) se establecen valores de distorsión permisible de entrepiso a fin de evitar el colapso de edificaciones estructuradas a base de muros de mampostería de carga. Dichos valores de distorsión permisible dependen del tipo de pieza (sólida o hueca) y de la presencia o ausencia de refuerzo horizontal. En la tabla 4 se presentan los valores límites establecidos en la normatividad. Como puede apreciarse, la normatividad no especifica si las piezas son del tipo artesanal, industrializado o de concreto.

Tabla 4. Distorsiones permisibles para seguridad contra colapso establecidas en el Apéndice A de las Normas Técnicas Complementarias para el Diseño por Sismo Edición 2004 (NTS, 2004).

\begin{tabular}{lc}
\hline \multicolumn{1}{c}{ Sistema estructural } & Distorsión [\%] \\
\hline $\begin{array}{l}\text { Muros de carga de mampostería de piezas macizas } \\
\text { con refuerzo horizontal o malla }\end{array}$ & 0.50 \\
$\begin{array}{l}\text { Muros de carga de mampostería de piezas macizas; } \\
\text { mampostería de piezas huecas confinada y reforzada }\end{array}$ & 0.40 \\
horizontalmente; o mampostería de piezas huecas \\
$\begin{array}{l}\text { confinada y reforzada con malla } \\
\text { Muros de carga de mampostería de piezas huecas } \\
\text { con refuerzo interior } \\
\begin{array}{l}\text { Muros de carga de mampostería que no cumplan con } \\
\text { las especificaciones para mampostería confinada ni } \\
\text { para mampostería reforzada interiormente }\end{array}\end{array}$ \\
\hline
\end{tabular}

Resulta de interés comparar los valores de distorsión permisible establecidos en la normatividad con los valores de distorsión asociados a las curvas de fragilidad desarrolladas para el estado de daño 2, el cual podría suponerse similar al estado límite de seguridad contra el colapso considerando que los muros de mampostería presentan una rápida pérdida de resistencia una vez alcanzada su capacidad ante cargas laterales. Por ejemplo, un valor de distorsión de $0.50 \%$, correspondiente a la primera categoría, tendría una probabilidad del orden de $25 \%$ de alcanzar o exceder el estado de daño 2 para muros construidos con tabique artesanal que incluyen acero de refuerzo horizontal. Sin embargo, si los muros son construidos con bloque de concreto y reforzados horizontalmente, para el mismo nivel de distorsión lateral se podría esperar una probabilidad de excedencia del $91 \%$.

Con respecto a la segunda categoría, el límite de distorsión permisible (0.40\%) excede en $29 \%$ a la tendencia central de la distorsión correspondiente al estado de daño $2(0.31 \%)$, asociado a muros de mampostería confinada con piezas sólidas, suponiendo que son de arcilla de barro recocido, sin refuerzo interior ensayados con y sin carga vertical de compresión, mientras que excede en aproximadamente $60 \%$ si solo se consideran muros ensayados en presencia de carga vertical de compresión. Por el contrario, el mismo nivel de distorsión permisible es muy cercano a la tendencia central de la distorsión $(0.39 \%)$ calculada para muros construidos con bloques de concreto sin refuerzo horizontal ensayados con y sin carga vertical de compresión. Sin embargo, el límite normativo de distorsión es inferior en un 53\% a la 
tendencia central de la distorsión (0.61\%) calculada para muros de mampostería construidos con piezas industrializada que incluyen acero de refuerzo horizontal (ensayados con y sin carga vertical).

Las observaciones anteriores sugieren que es importante especificar el tipo de pieza (tabique artesanal, tabique industrializado o bloque de concreto) al establecer los límites de distorsión permisible de entrepiso. Asimismo, sería deseable que los límites de distorsión lateral tuvieran la misma probabilidad de excedencia a fin de establecer criterios de desempeño uniforme. Por ello, las curvas de fragilidad y, particularmente, los valores de tendencia central y dispersión, presentadas en este estudio pueden ser una referencia para actualizar y calibrar los valores propuestos en el Apéndice A de la Normas Técnicas Complementarias para el Diseño por Sismo Edición 2004 (NTS, 2004).

\section{CONCLUSIONES Y RECOMENDACIONES}

En el estudio que se describe en este artículo se presentaron curvas de fragilidad basadas en distorsión lateral asociadas a dos estados de daño para muros de mampostería confinada construidos con tres tipos de pieza (tabique de barro recocido, tabique de arcilla industrializado y bloque de concreto). Las curvas de fragilidad se desarrollaron a partir de una base de datos que incluyó resultados experimentales provenientes de 118 especímenes de mampostería confinada ensayados en cinco países latinoamericanos. Derivado de este estudio, se ofrecen las conclusiones siguientes:

-Se observó que la dispersión en la estimación de la tendencia central de distorsiones asociadas a la aparición del primer agrietamiento diagonal y a la resistencia lateral en muros de mampostería confinada es significativa, como se aprecia en los resultados presentados en la tabla 5.

-La probabilidad de exceder un estado de daño en muros de mampostería para un nivel de distorsión lateral dado, expresada como curvas de fragilidad, depende del tipo de pieza, la inclusión del acero de refuerzo y, en menor medida, del nivel de carga axial de compresión actuante en el muro.

-La inclusión de varias fuentes de incertidumbre, tanto aleatoria como epistémica, tienen una influencia importante en la curvas de fragilidad basadas en distorsión lateral desarrolladas para muros de mampostería.

-Una comparación de los valores de distorsión permisible para edificaciones de mampostería establecidos en el Apéndice A de las Normas Técnicas Complementarias para el Diseño por Sismo con los valores de distorsión asociados a las curvas de fragilidad desarrolladas para el estado de daño 2 indica que es necesario una clasificación más detallada del tipo de pieza.

Las curvas de fragilidad derivadas de este estudio son una herramienta muy útil para establecer, o calibrar, valores de distorsión permisible para edificaciones de mampostería confinada en zonas sísmicas. Para tal fin, se recomienda considerar los valores descritos en la tabla 5. Asimismo, las curvas de fragilidad pueden emplearse en la estimación de pérdidas económicas en este tipo de edificaciones ante un escenario sísmico.

\section{AGRADECIMIENTOS}

El autor quisiera agradecer la participación del M.I. Miguel Negrete Padilla durante el desarrollo inicial de este estudio. Asimismo, dos revisores anónimos proporcionaron valiosos comentarios y 
sugerencias para la versión final de este artículo. Finalmente, se agradece a la Universidad Michoacana de San Nicolás de Hidalgo por las facilidades brindadas para el desarrollo de esta investigación.

\section{REFERENCIAS}

Aguilar, G, R Meli, R Díaz y R Vázquez-del-Mercado (1996), “Influence of horizontal reinforcement on the behavior of confined masonry walls", Eleventh World Conference on Earthquake Engineering, Acapulco, Paper No. 1380.

Alcocer, S M, J C Arias y L E Flores (2004), "Some developments in performance-based design of confined masonry structures”, Report PEER 2004/05, Pacific Earthquake Engineering Research Center 2004. pp. 233-244.

Alcocer, S M, y J A Zepeda (1999), "Behavior of multi-perforated clay brick walls under earthquake-type loading”, Eight North American Masonry Conference, Austin, Texas.

Alcocer, S A, J Ruiz, J Pineda y J A Zepeda (1996), "Retrofitting of confined masonry walls with wirewelded mesh”, Eleventh World Conference on Earthquake Eengineering, Acapulco, Paper No. 1471.

Alcocer, S M y R Meli (1995), "Test program on the seismic behavior of confined masonry walls”, The Masonry Society Journal, Vol. 13, pp.68-76.

Ang, A H-S, y W H Tang (2007), Probability concepts in engineering. Emphasis on applications to civil and environmental engineering, John Wiley \& Sons, New York.

Aslani, H, y E Miranda (2005), "Fragility assessment of slab-column connections in existing non-ductile reinforced concrete structures”, Journal of Earthquake Engineering, Vol. 9, No. 6, pp. 777-804.

Astroza, M, y A Schmidt (2004), "Capacidad de deformación de muros de albañilería confinada para distintos niveles de desempeño”, Revista de Ingeniería Sísmica, No. 70, pp. 59-75.

Carrillo, V, y A Molina (1997), "Evaluación del comportamiento de muros confinados de bloques macizos de arcilla con diferentes relaciones de aspecto ante cargas alternantes”, Tesis de Licenciatura, Univ. Central de Venezuela, Caracas.

Castilla, C (1998), "Evaluación de la respuesta de muros confinados de bloques de concreto contra ciclos severos de carga lateral”, Tesis de Doctorado, Univ. Central de Venezuela, Caracas.

Diez, J (1987), “Estudio experimental de muros de albañilería sometidos a carga lateral alternada”, Tesis de Licenciatura, Universidad de Chile, Santiago.

Dimova, S, y P Negro (2006), “Assessment of seismic fragility of structures with consideration of quality of construction”, Earthquake Spectra, 22(4): 909-936.

Earthquake Engineering Research Center (2006), “The Tecoman, Mexico earthquake January 21, 2003”, EERI-SMIS Reconnaissance Report.

Erberik, M A, y A S Elnashai (2003), "Seismic vulnerability of flat-slab structures”, Technical Report Mid-America Earthquake Center DS-9 Project, Univ. of Illinois at Urbana-Champaign.

Flores, L E (2003), "Evaluación experimental de marcos con muro diafragma de bloque, reforzados con recubrimiento de concreto", Tesis de Maestría, Div. de Estudios de Posgrado de la Fac. de Ing. Civil, Univ. Nacional Autónoma de México. 
Hernández, O, y R Meli (1976), "Modalidades de refuerzo para mejorar el comportamiento sísmico de muros de mampostería”, Reporte No 382, Instituto de Ingeniería, Univ. Nacional Autónoma de México.

Herrera, E (1992), "Efecto de la carga vertical en el comportamiento de muros de albañilería reforzada sometidos a carga lateral alternada", Tesis de Licenciatura, Universidad de Chile.

Meli, R (1975), “Comportamiento sísmico de muros de mampostería”, Reporte No 352, Instituto de Ingeniería, Univ. Nacional Autónoma de México.

Meli, R, y Hernández, O. (1975), "Efecto de hundimientos diferenciales en construcciones a base de muros de mampostería”, Reporte No 350, Instituto de Ingeniería, Univ. Nacional Autónoma de México.

Meli, R, y G Salgado (1969), “Comportamiento de muros de mampostería sujetos a carga lateral. Segundo informe”, Reporte No 237, Instituto de Ingeniería, Univ. Nacional Autónoma de México.

Meli, R, A Zeevaert y L Esteva (1968), “Comportamiento de muros de mampostería hueca ante carga lateral alternada”, Reporte No 156, Instituto de Ingeniería, Univ. Nacional Autónoma de México.

Muñoz, W (1992), "Estudio experimental del comportamiento de albañilería de bloques de hormigón sujetos a carga lateral alternada”, Tesis de Licenciatura, Universidad de Chile, Santiago.

NTS (2004), Normas Técnicas Complementarias para Diseño por Sismo, Gaceta oficial, Gobierno del Distrito Federal.

Pagni, C, y L Lowes (2006), "Fragility functions for older reinforced concrete joints", Earthquake Spectra, Vol. 22, No. 1, pp. 215-238.

Reyes, C y R Meli (1999), "Relaciones distorsión de entrepiso-daño y velocidades y aceleraciones de piso con incomodidad personal y daño a objetos”, Memorias del XII Congreso Nacional de Ingeniería Sísmica, Morelia, Michoacán, pp. 992-1001.

Rodríguez, M (2005), "Design and evaluation of masonry dwellings in seismic zones”, Earthquake Spectra, Vol. 21, No. 2, pp. 465-492.

Ruiz, J y M Negrete (2007), "Evaluación sísmica de estructuras de mampostería confinada basada en su desempeño estructural”, Memorias del XVI Congreso Nacional de Ingeniería Sísmica, Sociedad Mexicana de Ingeniería Sísmica, Ixtapa-Zihuatanejo, Artículo XII-08.

Ruiz, J, T Sánchez y E Miranda (2003), “Capítulo 6: Observaciones sobre el comportamiento y diseño de edificaciones de mampostería en zonas sísmicas”, en: Edificaciones de mampostería para vivienda, Fundación ICA, Sociedad Mexicana de Ingeniería Estructural, 3ª. Edición.

Ruiz, J, y S M Alcocer (1998), "Desempeño experimental de estructuras de mampostería confinada rehabilitadas mediante el uso de malla de alambre”, Revista de Ingeniería Sísmica, No. 59, pp. 5979.

San Bartolomé, A (1994), "Masonry construction, Seismic behavior and structural design (in Spanish), Pontificia Universidad Católica del Perú, Fondo Editorial 1994, 228 pp.

Terán, A y O Zúñiga (2007), "Evaluación estructural basada en desplazamientos para edificaciones de mampostería confinada, el desarrollo mexicano”, Memorias del $5^{\circ}$. Simposio Nacional de Ingeniería Estructural en la Vivienda, Querétaro, Qro.

Treviño, E, S M Alcocer, L E Flores, R Larrúa, J M Zárate y L Gallegos (2004), "Investigación experimental del comportamiento de muros de mampostería confinada de bloques de concreto 
sometidos a cargas laterales cíclicas reversibles reforzados con acero grados 60 y 42”, Memorias del XIV Congreso Nacional de Ingeniería Estructural, Acapulco.

Wen, Y K, y B R Ellingwood (2005), "The role of fragility in consequence-based engineering”, Earthquake Spectra, Vol. 21, No. 3, pp.861-877.

Wen, Y K, B R Ellingwood, J Bracci (2004), "Vulnerability function framework for consequence-based engineering”, Report DS-4, Mid-America Earthquake Center, University of Illinois at UrbanaChampaign.

Yamín, L E, L García, J Galeano y G Reyes (1993), "Estudio del comportamiento de muros de mampostería confinada sometidos a cargas verticales y horizontales simultáneas”, Décimas Jornadas Estructurales de la Ingeniería en Colombia.

Zepeda, J A, S M Alcocer y L E Flores (2000), "Earthquake-resistant construction with multi-perforated clay brick walls”, Twelfth World Conference on Earthquake Engineering; Auckland, Paper No. 1541. 
Evaluación de la fragilidad basada en distorsión lateral para muros de mampostería confinada

\section{APENDICE A}

Tabla A.1. Información de muros de mampostería confinada construidos con piezas macizas de barro artesanal.

\begin{tabular}{|c|c|c|c|c|c|c|c|c|c|}
\hline Referencia & $\begin{array}{l}\text { Nombre del } \\
\text { espécimen }\end{array}$ & $\begin{array}{c}\mathrm{v}_{\mathrm{m}} \\
{[\mathrm{kg} / \mathrm{cm} 2]}\end{array}$ & $\begin{array}{c}\mathrm{H} / \mathrm{L} \\
{[\mathrm{cm} / \mathrm{cm}]}\end{array}$ & $\begin{array}{l}\rho_{h} \\
{[\%]}\end{array}$ & $\begin{array}{c}\sigma_{v} \\
{[\mathrm{~kg} / \mathrm{cm}} \\
2]\end{array}$ & ${\underset{[\%]}{\delta_{a g r}}(+)}$ & $\delta_{\text {max }}(+)$ & $\underbrace{\delta_{a g r}}_{[\%]}$ & $\delta_{\text {max }}(-)$ \\
\hline \multirow{5}{*}{$\begin{array}{l}\text { Meli y } \\
\text { Hernández } \\
\text { (1975) }\end{array}$} & & & & & & & & & \\
\hline & 11 & 4.90 & 0.90 & $*$ & $*$ & 0.165 & $*$ & $*$ & * \\
\hline & 21 & 4.90 & 0.80 & $*$ & $*$ & 0.110 & $*$ & $*$ & * \\
\hline & 81 & 4.90 & 0.90 & $*$ & $*$ & 0.060 & $*$ & $*$ & $*$ \\
\hline & 141 & 6.20 & 0.90 & $*$ & $*$ & 0.050 & $*$ & $*$ & $*$ \\
\hline \multirow{5}{*}{$\begin{array}{l}\text { Herrera (1992), } \\
\text { en Astroza y } \\
\text { Schmidt (2004) }\end{array}$} & B11 & 6.67 & 1.00 & $*$ & * & 0.060 & 0.100 & 0.070 & 0.260 \\
\hline & B12 & 2.35 & 1.00 & $*$ & 4.76 & 0.150 & 0.280 & 0.240 & 0.360 \\
\hline & B13 & 2.35 & 1.00 & $*$ & 4.76 & 0.120 & 0.180 & 0.130 & 0.190 \\
\hline & B14 & 2.35 & 1.00 & $*$ & 4.76 & 0.110 & 0.460 & 0.110 & 0.500 \\
\hline & B2 & 2.35 & 1.00 & $*$ & 2.38 & 0.240 & 0.460 & 0.240 & 0.620 \\
\hline \multirow{2}{*}{$\begin{array}{c}\text { Yamin et al. } \\
\text { (1993) }\end{array}$} & & 1.94 & & 0.11 & & & & & \\
\hline & 1 & & 1.00 & 0 & $*$ & 0.330 & 0.520 & $*$ & * \\
\hline \multirow{3}{*}{$\begin{array}{c}\text { Alcocer y Meli } \\
\text { (1995) }\end{array}$} & WBW & 10.0 & 1.67 & * & 5.00 & 0.120 & 0.600 & 0.120 & 0.350 \\
\hline & W-W & 10.0 & 1.67 & $*$ & 5.00 & 0.100 & 1.000 & 0.120 & 0.600 \\
\hline & WWW & 10.0 & 1.67 & $*$ & 5.00 & 0.071 & 0.400 & 0.053 & 0.410 \\
\hline \multirow{7}{*}{$\begin{array}{l}\text { Alcocer et al. } \\
\text { (1996) }\end{array}$} & $3 \mathrm{D}$ & 3.90 & 1.60 & $*$ & 5.00 & 0.127 & 0.357 & 0.080 & 0.307 \\
\hline & & 7.50 & & 0.07 & & & & & \\
\hline & M-072 & & 1.00 & 1 & 5.00 & 0.200 & 0.602 & 0.107 & 0.602 \\
\hline & & 7.50 & & 0.14 & & & & & \\
\hline & M-147 & & 1.00 & 7 & 5.00 & 0.180 & 0.393 & 0.163 & 0.370 \\
\hline & & 7.50 & & 0.21 & & & & & \\
\hline & M-211 & & 1.00 & 1 & 5.00 & 0.200 & 0.600 & 0.200 & 0.600 \\
\hline \multirow{11}{*}{$\begin{array}{l}\text { Aguilar et al } \\
\text { (1996) }\end{array}$} & & 7.50 & & 0.09 & & & & & \\
\hline & WBW-B & & 1.60 & 1 & 5.00 & 0.190 & 0.7530 & 0.099 & 0.588 \\
\hline & & 6.50 & & 0.10 & & & & & \\
\hline & WBW-E & & 1.60 & 2 & 5.00 & 0.108 & 0.2210 & 0.141 & 0.500 \\
\hline & M-0-E6 & 7.50 & 1.00 & $*$ & 5.00 & 0.134 & 0.455 & 0.100 & 0.415 \\
\hline & & 5.70 & & 0.21 & & & & & \\
\hline & M-3/8-Z6 & & 1.00 & 1 & 5.00 & 0.2207 & 0.5654 & 0.1847 & 0.5809 \\
\hline & & 4.00 & & 0.19 & & & & & \\
\hline & M-1/4-E6 & & 1.00 & 0 & 5.00 & 0.1179 & 0.8268 & 0.1628 & 0.5979 \\
\hline & & 3.80 & & 0.07 & & & & & \\
\hline & M-5/32-E20 & & 1.00 & 1 & 5.00 & 0.1683 & 0.6057 & 0.1673 & 0.5438 \\
\hline \multirow{10}{*}{$\begin{array}{c}\text { Carrillo y } \\
\text { Molina (1997) } \\
\text { en Astroza y } \\
\text { Schmidt (2004) }\end{array}$} & Muro 1 & 6.61 & 0.71 & $*$ & $*$ & 0.070 & 0.080 & 0.080 & 0.080 \\
\hline & Muro 10 & 6.61 & 0.95 & $*$ & $*$ & 0.050 & 0.560 & 0.170 & 0.730 \\
\hline & Muro 2 & 6.61 & 0.71 & $*$ & 9.28 & 0.050 & 0.280 & 0.050 & 0.290 \\
\hline & Muro 3 & 6.61 & 0.71 & $*$ & 4.64 & 0.070 & 0.090 & 0.080 & 0.110 \\
\hline & Muro 4 & 6.61 & 1.13 & $*$ & 6.96 & 0.070 & 0.460 & 0.070 & 0.130 \\
\hline & Muro 5 & 6.61 & 1.13 & $*$ & $*$ & 0.070 & 0.730 & 0.070 & 0.730 \\
\hline & Muro 6 & 6.61 & 1.13 & $*$ & 13.92 & 0.070 & 0.150 & 0.040 & 0.290 \\
\hline & Muro 7 & 6.61 & 0.95 & * & 13.92 & 0.070 & 0.360 & 0.070 & 0.440 \\
\hline & Muro 8 & 6.61 & 0.95 & $*$ & $*$ & 0.070 & 0.140 & 0.070 & 0.220 \\
\hline & Muro 9 & 6.61 & 0.95 & $*$ & 4.64 & 0.060 & 0.870 & 0.150 & 0.650 \\
\hline
\end{tabular}

*Información no disponible

${ }^{1}$ Carga monótona 
Tabla A2. Información de muros de mampostería confinada construidos con piezas industrializadas de arcilla (sólidas o multiperforadas).

\begin{tabular}{|c|c|c|c|c|c|c|c|c|c|}
\hline Referencia & $\begin{array}{l}\text { Nombre del } \\
\text { espécimen }\end{array}$ & $\begin{array}{c}v_{m} \\
{\left[\mathrm{~kg} / \mathrm{cm}^{2}\right]}\end{array}$ & $\begin{array}{c}H / L \\
{[\mathrm{~cm} / \mathrm{cm}]}\end{array}$ & $\begin{array}{l}\rho_{h} \\
{[\%]}\end{array}$ & $\begin{array}{c}\sigma_{v} \\
{\left[\mathrm{~kg} / \mathrm{cm}^{2}\right]}\end{array}$ & $\begin{array}{c}\delta_{a g r}(+) \\
{[\%]}\end{array}$ & $\delta_{\max }(+)$ & $\begin{array}{c}\delta_{a g r}(-) \\
{[\%]}\end{array}$ & $\begin{array}{c}\delta_{\max }(-) \\
{[\%]}\end{array}$ \\
\hline \multirow{2}{*}{$\begin{array}{c}\text { Meli y Salgado } \\
\text { (1969) }\end{array}$} & $901^{a}$ & 7.0 & 0.96 & * & $*$ & 0.085 & 0.085 & 0.192 & 0.590 \\
\hline & $902^{\mathrm{a}}$ & 7.0 & 0.96 & $*$ & 4.81 & 0.395 & 0.360 & 0.443 & 0.740 \\
\hline \multirow{8}{*}{$\begin{array}{l}\text { Hernández y } \\
\text { Meli (1976) }\end{array}$} & $1^{\mathrm{b}}$ & 3.9 & 1.00 & $*$ & $*$ & 0.100 & 1.050 & $*$ & $*$ \\
\hline & $2^{\mathrm{b}}$ & 3.9 & 1.00 & 0.059 & $*$ & 0.065 & 1.400 & $*$ & $*$ \\
\hline & $3^{b}$ & 3.9 & 1.00 & 0.059 & $*$ & 0.070 & 2.250 & * & $*$ \\
\hline & $4^{b}$ & 3.9 & 1.00 & 0.059 & $*$ & 0.055 & 0.650 & * & $*$ \\
\hline & $5^{b}$ & 3.9 & 1.00 & 0.098 & $*$ & 0.040 & 1.650 & $*$ & $*$ \\
\hline & $6^{b}$ & 3.9 & 1.00 & 0.059 & * & 0.065 & 0.900 & * & $*$ \\
\hline & $7^{b}$ & 3.9 & 1.00 & * & $*$ & 0.073 & 0.140 & * & $*$ \\
\hline & $8^{b}$ & 3.9 & 1.00 & 0.049 & $*$ & 0.035 & 0.440 & $*$ & $*$ \\
\hline \multirow{5}{*}{$\begin{array}{l}\text { Herrera (1987), } \\
\text { en Astroza y } \\
\text { Schmidt (2004) }\end{array}$} & $\mathrm{A} 11^{\mathrm{a}}$ & 8.0 & 1.00 & $*$ & $*$ & 0.090 & 0.210 & 0.100 & 0.360 \\
\hline & $\mathrm{A} 12^{\mathrm{a}}$ & 8.0 & 1.00 & $*$ & 2.38 & 0.350 & 0.350 & 0.330 & 0.420 \\
\hline & $\mathrm{A} 13^{\mathrm{a}}$ & 8.0 & 1.00 & $*$ & 4.76 & 0.180 & 0.180 & 0.190 & 0.190 \\
\hline & $\mathrm{A} 14^{\mathrm{a}}$ & 8.0 & 1.00 & $*$ & 4.76 & 0.050 & 0.210 & 0.060 & 0.160 \\
\hline & $\mathrm{A} 2^{\mathrm{a}}$ & 8.0 & 1.00 & $*$ & 2.38 & 0.160 & 0.160 & 0.130 & 0.130 \\
\hline \multirow{2}{*}{$\begin{array}{c}\text { Diez (1992), } \\
\text { en Astroza y } \\
\text { Schmidt (2004) }\end{array}$} & $\operatorname{MRG} 1^{\mathrm{a}}$ & 10.56 & 1.00 & $*$ & * & 0.040 & 0.260 & 0.050 & 0.320 \\
\hline & $\operatorname{MRG} 2^{\mathrm{a}}$ & 10.56 & 1.00 & $*$ & $*$ & 0.030 & 0.730 & 0.010 & 0.290 \\
\hline \multirow{2}{*}{$\begin{array}{c}\text { Yamin et al. } \\
\text { (1993) }\end{array}$} & & 2.24 & & & & & & & \\
\hline & 2 & & - & $*$ & $*$ & 0.470 & 0.550 & $*$ & $*$ \\
\hline \multirow{9}{*}{$\begin{array}{l}\text { San Bartolome } \\
\text { (1994) }\end{array}$} & MR1 & 8.76 & 1.00 & $*$ & $*$ & 0.070 & 0.621 & * & $*$ \\
\hline & MR3 & 8.76 & 1.00 & 0.1600 & $*$ & 0.057 & 0.171 & * & $*$ \\
\hline & MR4 & 8.76 & 1.00 & 0.1600 & $*$ & 0.060 & 0.508 & * & $*$ \\
\hline & MR5 & 8.76 & 1.00 & 0.0800 & $*$ & 0.043 & 0.613 & * & $*$ \\
\hline & MV1 & 8.76 & 1.07 & * & $*$ & 0.166 & 0.683 & * & $*$ \\
\hline & MV2 & 8.76 & 1.07 & $*$ & 1.72 & 0.142 & 0.509 & * & $*$ \\
\hline & MV3 & 8.76 & 1.07 & $*$ & 5.20 & 0.164 & 0.213 & * & $*$ \\
\hline & MV4 & 8.76 & 1.07 & $*$ & 5.20 & 0.108 & 0.630 & * & $*$ \\
\hline & MV5 & 8.76 & 1.07 & $*$ & 8.60 & 0.107 & 0.435 & $*$ & $*$ \\
\hline \multirow{4}{*}{$\begin{array}{c}\text { Alcocer y } \\
\text { Zepeda (1999) }\end{array}$} & $\mathrm{N} 1^{\mathrm{b}}$ & 10.40 & 1.00 & * & $*$ & 0.030 & 0.730 & 0.010 & 0.290 \\
\hline & $\mathrm{N} 2^{\mathrm{b}}$ & 10.10 & 1.00 & $*$ & 4.00 & 0.097 & 0.240 & 0.091 & 0.229 \\
\hline & N3 ${ }^{b}$ & 12.10 & 1.00 & 0.0491 & 4.00 & 0.081 & 0.500 & 0.070 & 0.354 \\
\hline & $\mathrm{N} 4^{\mathrm{b}}$ & 7.5 & 1.00 & 0.1845 & 4.00 & 0.100 & 0.600 & 0.100 & 0.601 \\
\hline
\end{tabular}


Evaluación de la fragilidad basada en distorsión lateral para muros de mampostería confinada

Tabla A3. Información de muros de mampostería confinada construidos con bloques de concreto industrializados.

\begin{tabular}{|c|c|c|c|c|c|c|c|c|c|}
\hline Referencia & $\begin{array}{l}\text { Nombre del } \\
\text { espécimen }\end{array}$ & $\begin{array}{c}v_{m} \\
{\left[\mathrm{~kg} / \mathrm{cm}^{2}\right]}\end{array}$ & $\begin{array}{c}H / L \\
{[\mathrm{~cm} / \mathrm{cm}]}\end{array}$ & $\begin{array}{l}\rho_{h} \\
{[\%]}\end{array}$ & $\begin{array}{c}\sigma_{V} \\
{\left[\mathrm{~kg} / \mathrm{cm}^{2}\right]}\end{array}$ & $\begin{array}{c}\delta_{a g r} \\
(+) \\
{[\%]} \\
\end{array}$ & $\delta_{\text {max }}(+)$ & $\begin{array}{c}\delta_{\text {agr }}(-) \\
{[\%]}\end{array}$ & $\begin{array}{c}\delta_{\max }(-) \\
{[\%]}\end{array}$ \\
\hline \multirow{15}{*}{$\begin{array}{c}\text { Meli et } \\
\text { al., (1968) }\end{array}$} & $301^{b}$ & $*$ & 0.98 & * & * & 0.060 & 0.250 & $*$ & $*$ \\
\hline & $302^{b}$ & $*$ & 0.90 & $*$ & $*$ & 0.090 & 0.100 & $*$ & $*$ \\
\hline & $303^{b}$ & $*$ & 0.90 & $*$ & $*$ & 0.080 & 0.210 & $*$ & $*$ \\
\hline & $304^{b}$ & $*$ & 0.90 & 0.237 & $*$ & 0.100 & 0.210 & $*$ & $*$ \\
\hline & $307^{b}$ & $*$ & 0.90 & 0.020 & $*$ & 0.100 & 0.150 & $*$ & $*$ \\
\hline & $308^{b}$ & $*$ & 0.90 & 0.016 & 4.17 & 0.040 & 0.270 & $*$ & $*$ \\
\hline & $309^{b}$ & $*$ & 0.90 & 0.016 & $*$ & 0.160 & 0.290 & $*$ & $*$ \\
\hline & $311^{\mathrm{b}}$ & $*$ & 0.90 & 0.016 & 2.08 & 0.220 & 0.340 & $*$ & $*$ \\
\hline & $312^{b}$ & $*$ & 0.90 & 0.016 & $*$ & 0.300 & 0.300 & $*$ & $*$ \\
\hline & $313^{b}$ & $*$ & 0.90 & 0.016 & $*$ & 0.230 & 0.470 & $*$ & $*$ \\
\hline & $314^{\mathrm{b}}$ & $*$ & 0.90 & 0.016 & 2.08 & 0.160 & 0.460 & $*$ & $*$ \\
\hline & $315^{b}$ & $*$ & 0.90 & $*$ & $*$ & 0.100 & 0.130 & $*$ & $*$ \\
\hline & $316^{\mathrm{b}}$ & $*$ & 0.90 & $*$ & 4.17 & 0.210 & 0.270 & $*$ & $*$ \\
\hline & $317^{b}$ & $*$ & 0.90 & 0.016 & $*$ & 0.260 & 0.300 & $*$ & $*$ \\
\hline & $318^{\mathrm{b}}$ & $*$ & 0.90 & 0.016 & $*$ & 0.090 & 0.250 & $*$ & $*$ \\
\hline \multirow{10}{*}{$\begin{array}{c}\text { Meli y } \\
\text { Salgado } \\
\text { (1969) }\end{array}$} & $401^{b}$ & 5.80 & 1.04 & $*$ & $*$ & 0.221 & 0.420 & 0.228 & 0.435 \\
\hline & $402^{b}$ & 5.80 & 1.04 & $*$ & 2.47 & 0.050 & 0.280 & 0.106 & 0.239 \\
\hline & $403^{b}$ & 5.80 & 1.04 & $*$ & $*$ & 0.150 & 0.260 & 0.187 & 0.401 \\
\hline & $404^{b}$ & 5.80 & 1.04 & $*$ & $*$ & 0.202 & 0.294 & 0.283 & 0.515 \\
\hline & $405^{b}$ & 5.80 & 1.04 & $*$ & $*$ & 0.172 & 0.453 & 0.285 & 0.164 \\
\hline & $406^{\mathrm{b}}$ & 5.80 & 1.04 & $*$ & 3.70 & 0.191 & 0.501 & 0.298 & 0.544 \\
\hline & $407^{b}$ & 5.80 & 1.04 & $*$ & $*$ & 0.072 & 0.392 & 0.156 & 0.240 \\
\hline & $408^{\mathrm{b}}$ & 5.80 & 1.04 & $*$ & 3.70 & 0.177 & 0.304 & 0.256 & 0.366 \\
\hline & $409^{b}$ & 5.80 & 1.04 & $*$ & $*$ & 0.137 & 0.212 & 0.044 & 0.164 \\
\hline & $410^{\mathrm{b}}$ & 5.80 & 1.04 & $*$ & 7.40 & 0.076 & 0.154 & 0.123 & 0.127 \\
\hline \multirow{7}{*}{$\begin{array}{l}\text { Hernández } \\
\text { y Meli } \\
\text { (1976) }\end{array}$} & $9^{b}$ & 3.75 & 1.00 & 0.0412 & $*$ & 0.030 & 0.350 & $*$ & $*$ \\
\hline & $10^{\mathrm{b}}$ & 3.75 & 1.00 & $*$ & $*$ & 0.046 & 0.650 & $*$ & $*$ \\
\hline & $11^{\mathrm{b}}$ & 3.75 & 1.00 & $*$ & $*$ & 0.086 & 0.250 & $*$ & $*$ \\
\hline & $12^{\mathrm{b}}$ & 4.50 & 1.00 & $*$ & $*$ & 0.045 & 0.550 & $*$ & $*$ \\
\hline & $13^{b}$ & 4.50 & 1.00 & $*$ & $*$ & 0.080 & 0.500 & $*$ & $*$ \\
\hline & $14^{\mathrm{b}}$ & 3.45 & 1.00 & 0.0412 & $*$ & 0.028 & 0.550 & $*$ & $*$ \\
\hline & $15^{\mathrm{b}}$ & 3.45 & 1.00 & $*$ & $*$ & 0.040 & 0.600 & $*$ & $*$ \\
\hline \multirow{5}{*}{$\begin{array}{c}\text { Muñoz } \\
\text { (1992), } \\
\text { Astroza y } \\
\text { Schmidt } \\
\text { (2004) }\end{array}$} & $\mathrm{C} 11^{\mathrm{a}}$ & 7.10 & 1.00 & * & 4.17 & 0.140 & 0.510 & 0.230 & 0.500 \\
\hline & $\mathrm{C} 12^{\mathrm{a}}$ & 7.10 & 1.00 & $*$ & 4.17 & 0.130 & 0.390 & 0.230 & 0.610 \\
\hline & $\mathrm{C} 13^{\mathrm{a}}$ & 7.10 & 1.00 & $*$ & 4.17 & 0.140 & 0.380 & 0.140 & 0.430 \\
\hline & & & & & & & & & \\
\hline & $\mathrm{C} 14^{\mathrm{a}}$ & 7.10 & 1.00 & $*$ & $*$ & 0.120 & 0.620 & 0.120 & 0.500 \\
\hline \multirow{9}{*}{$\begin{array}{l}\text { Castilla } \\
\text { (1998), } \\
\text { en Astroza } \\
\text { y Schmidt } \\
\text { (2004) }\end{array}$} & Muro $1^{\mathrm{a}}$ & 7.64 & 0.97 & * & 7.68 & 0.160 & 0.390 & 0.050 & 0.460 \\
\hline & Muro $2^{a}$ & 7.64 & 0.97 & $*$ & 3.89 & 0.100 & 0.380 & 0.040 & 0.430 \\
\hline & Muro $3^{a}$ & 7.64 & 0.97 & * & $*$ & 0.130 & 0.680 & 0.040 & 0.670 \\
\hline & Muro $4^{\mathrm{a}}$ & 7.64 & 0.74 & $*$ & 5.84 & 0.060 & 0.310 & 0.060 & 0.310 \\
\hline & Muro $5^{a}$ & 7.64 & 0.74 & $*$ & $*$ & 0.040 & 0.170 & 0.040 & 0.210 \\
\hline & Muro $6^{\mathrm{a}}$ & 7.64 & 0.74 & $*$ & 3.89 & 0.020 & 0.250 & 0.020 & 0.350 \\
\hline & Muro $7^{\mathrm{a}}$ & 7.64 & 1.26 & $*$ & 7.68 & 0.130 & 0.670 & 0.250 & 0.700 \\
\hline & Muro $8^{\mathrm{a}}$ & 7.64 & 1.26 & $*$ & $*$ & 0.030 & 0.830 & 0.060 & 0.830 \\
\hline & Muro $9^{a}$ & 7.64 & 1.26 & $*$ & 3.89 & 0.030 & 0.750 & 0.080 & 0.710 \\
\hline $\begin{array}{l}\text { Flores } \\
(2003)\end{array}$ & $\mathrm{T}^{\mathrm{c}}$ & 2.61 & 0.96 & $*$ & $*$ & 0.030 & 0.601 & 0.100 & 0.607 \\
\hline \multirow{8}{*}{$\begin{array}{l}\text { Treviño } \\
\text { et. al. } \\
\text { (2004) }\end{array}$} & $421^{c}$ & 4.31 & 0.98 & * & 5.50 & 0.080 & $*$ & $*$ & $*$ \\
\hline & $422^{c}$ & 4.31 & 0.98 & $*$ & 5.50 & 0.100 & $*$ & $*$ & $*$ \\
\hline & $423^{c}$ & 4.31 & 0.98 & $*$ & 5.50 & 0.080 & $*$ & $*$ & $*$ \\
\hline & $424^{c}$ & 4.31 & 0.98 & $*$ & 5.50 & 0.070 & $*$ & $*$ & $*$ \\
\hline & $601^{\mathrm{c}}$ & 4.31 & 0.98 & $*$ & 5.50 & 0.100 & $*$ & $*$ & $*$ \\
\hline & $602^{\mathrm{c}}$ & 4.31 & 0.98 & $*$ & 5.50 & 0.150 & $*$ & $*$ & $*$ \\
\hline & $603^{c}$ & 4.31 & 0.98 & $*$ & 5.50 & 0.070 & $*$ & $*$ & $*$ \\
\hline & $604^{c}$ & 4.31 & 0.98 & $*$ & 5.50 & 0.110 & $*$ & $*$ & $*$ \\
\hline
\end{tabular}

